\title{
The Design and Implementation of Database on Library Management Information System
}

\author{
Hui Zhai \\ Department of Information Engineering, Henan Polytechnic \\ Zhengzhou, Henan, China 450046 \\ e-mail: hnzh1121@163.com
}

\author{
Hui Shi \\ Department of Information Engineering, Henan Polytechnic \\ Zhengzhou, Henan, China 450046
}

\author{
Rui Zhai \\ South No.1 community, Yungang streets, Fengtai \\ Beijing, China 100074 \\ e-mail: zk_rui@163.com
}

\begin{abstract}
The stand or fall of database design was related to an information management system was really practical. Analysis of user demand from the library management information system, the design and implementation of the database part in the system development process was discussed as a key point, and the concrete realization link was analyzed and discussed also.
\end{abstract}

Keywords-Management information system; database; design; Entity-Relationship

\section{INTRODUCTION}

The library played an important role in the daily teaching, scientific research and learning among teachers and students, and to promote its informatization was the powerful guarantee of school education, teaching and scientific research. ${ }^{[1]}$ The management of the library books information by using computer could reduce manual management mistakes and enhance the efficiency of book management greatly. It could ensure the integrity of the books information and speed up the turnover of the books resources. Therefore, using computer to manage library books information had very important significance. The database was the data integrated that was involved in a enterprise, an organization or a department. The database not only reflected the content of the data itself and the connection between the response data. ${ }^{[2][3]}$ Database design ${ }^{[4]}$ was to establish a database and its application system technology .It was the core technology of the information system development and construction. Specifically, the database design was to establish database and its application system for a given application environment in order to be able to stored data effectively and meet all kinds of users's the application requirements (information requirements and processing requirements). The core of library management information system was actually how to use and operation database. In addition,we should pay attention to standard naming for design, maintenance and inquires especially in the database design. ${ }^{[5]}$ As for the system development, SQL Server 2005 could be chosen for the system to create the background database.
II. THE DESCRIPTION OF SYSTEM FUNCTION

This system was designed for the library information management of school, the usage was mainly for processing the daily borrowing, returning and various query operation. After analysis the requirement of system, it had been made clear that the system must realize some function mainly as the following several aspects:

(1) Books borrowing treatment: loan processing, returning processing;

(2) Information query: books information query, borrowing information query, readers information query;

(3) Book information management: The coding warehousing of books, the modification and delete of book information, modify and delete of book information;

(4) Readers' information management: the readers' information to add, modify, and delete;

(5) Employee's information management: the employee's information to add, modify, and delete;

(6) System Settings: management user password, login management, set up the reader category.

\section{The Design AND IMPLEMENTATION OF ThE DATABASE}

Database design mainly included requirement analysis, concept structure design stage, the logic structure design stage,physical structure design stage,database implementation stage, database operation and maintenance stage, there're six steps altogether. Among them;Conceptual structure design was the user's data needs which was got in demand analysis stage to be synthesize and abstracted, then turn them into a unified conceptual model. It was mainly expressed by the Entity-Relationship model;Logical structure design was to make sure the logical structure of database, it was to turn E-R model which was got in the concept structure design stage into relation model;Physical structure design stage was to choose a logical data model which was the most suitable for applications environment for physical structure (including storage structure and access method). ${ }^{[6]}$ 


\section{A.Concept Structure Design}

Through analyzing the function of the library management information system, we could draw a conclusion that was an entity of a system mainly includes books information entity, readers' information entity, borrowing information entity and employee information entity, etc.

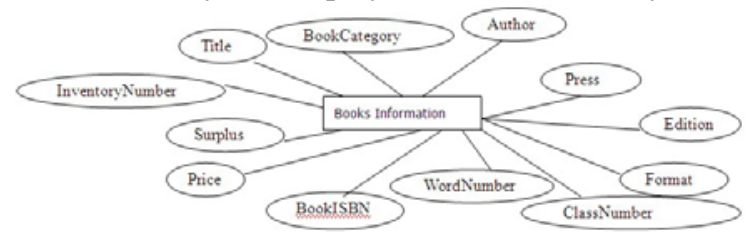

Figure 1. Books Information Entity E-R Diagram

Description:the book information entity recorded mainly the basic information of a book to get data which was to meet the needs of management. Among them, the "BookISBN" was an only standard Numbers of a legal publication. It was considered as the entity of the primary key. "Inventory Number" refered to how many books there were in the library. "Surplus" refered to how many books could be lent out yet.

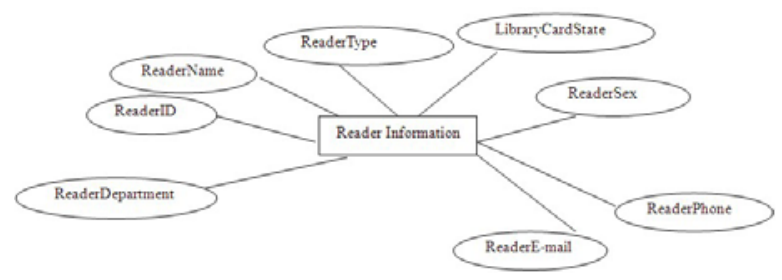

Figure 2. Reader Information E-R Diagram

Description:the reader information entity mainly recorded the reader's personal information and his borrowing state information from the library .Among them,"ReaderID" was the main key. "LibraryCardState" displayed if you could borrow books now. "ReaderType" showed the reader was a teacher, student or a worker.

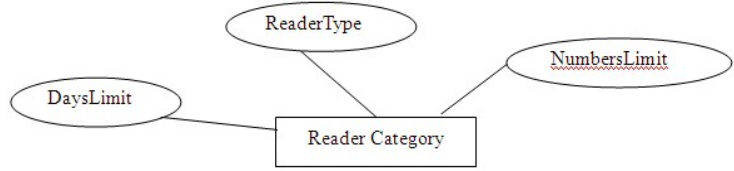

Figure 3. Reader Category E-R Diagram

Description:It was used to set up the restrictions about borrowing books among different category readers. Among them, "ReaderType" was the main key. "DaysLimit" showed how many days could be borrowed. "NumberLimit" showed the number of books that could be borrowed.

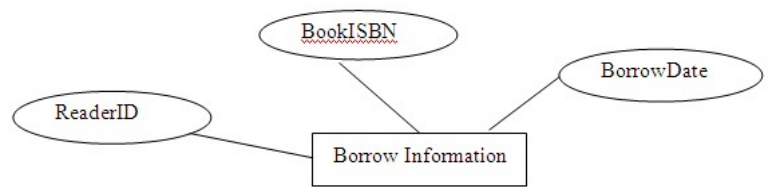

Figure 4. Borrow Information E-R Diagram

Description:The entity recorded which readers borrowed the book and what the date was. Among them,"BorrowDate" said the date of lending book.

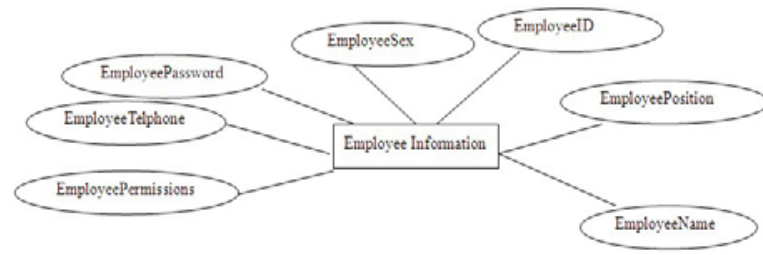

Figure 5. Employee Information E-R Diagram

Description:The entity said library staff's information. Among them, “EmployeeID” was employee numbers ,and it was primary key;"EmployeePwd” was login password 。 "EmployeePermissions" was used to determine that the employee could use which parts of function of the system.

\section{B.Logic Structure Design}

Turned above E-R diagram into relation model :

(1) Book(BookISBN 、BookClaNum 、 BookTitle 、 BookCategory 、BookAuthor、BookPress、BookEdition、 BookFormat 、BookWordNum 、BookPrice 、 BookInvNum、BookSur);

(2) Reader(ReaderID 、 ReaderName 、 ReaderType 、 LibraryCardState 、 ReaderSex 、 ReaderDepartment 、 ReaderPhone、ReaderEmail);

(3) ReaderCategory(ReaderType 、 NumbersLimit 、 DaysLimit);

(4) Borrow(ReaderID、BookISBN、BorrowDate);

(5) Employee(EmpID 、 EmpName 、 EmpSex 、 EmpPosition、EmpTel、EmpPwd、EmpPermissions);

\section{The Realization of Database}

According to all of above analysis and design system database, the lists was main used as follows:

\begin{tabular}{|c|c|c|c|}
\hline & Column Hame & Data Type & Allow Empty \\
\hline & BookISBY & char (20) & $\square$ \\
\hline & BookClatium & char (20) & $\square$ \\
\hline & BookTitle & char (30) & $\square$ \\
\hline & BookCategory & char (20) & $\square$ \\
\hline & Bookkuthor & char (20) & $\square$ \\
\hline & BookPress & char (20) & $\square$ \\
\hline & Bookfditi on & $\operatorname{char}(10)$ & $\square$ \\
\hline & Bookf ormat & char (10) & $\square$ \\
\hline & Bookth or dNum & $\operatorname{char}(10)$ & $\square$ \\
\hline & BookPrice & float & $\square$ \\
\hline & BookInvilum & int & $\square$ \\
\hline & Booksur & int & $\square$ \\
\hline \multicolumn{4}{|c|}{ Figure 6. Books Information Table } \\
\hline & Column Hame & Data Type & Allow Empty \\
\hline 8 & ReaderID & $\operatorname{char}(10)$ & $\square$ \\
\hline & ReaderHame & $\operatorname{char}(10)$ & $\square$ \\
\hline & ReaderType & $\operatorname{char}(10)$ & $\square$ \\
\hline & LibraryCardState & $\operatorname{char}(10)$ & $\square$ \\
\hline & ReaderSex & char (2) & $\square$ \\
\hline & ReaderDepartment & $\operatorname{char}(30)$ & $\square$ \\
\hline & ReaderPhone & char (20) & $\square$ \\
\hline & ReaderEmail & char (20) & $\square$ \\
\hline
\end{tabular}

Figure 7. Reader Information Table 


\begin{tabular}{|c|c|c|c|}
\hline & Column Hame & Data Type & Allow Empty \\
\hline \multirow[t]{5}{*}{8} & ReaderType & char $(10)$ & $\square$ \\
\hline & HumbersLimit & int & $\square$ \\
\hline & DaysLimit & int & $\square$ \\
\hline & \multicolumn{3}{|c|}{ Figure 8. Reader Category Table } \\
\hline & Column Hame & Data Type & Allow Empty \\
\hline ? & ReaderID & char (10) & $\square$ \\
\hline 8 & BookISBN & char (20) & $\square$ \\
\hline & BorrowDate & datetime & $\square$ \\
\hline \multicolumn{4}{|c|}{ Figure 9. Borrow Information Table } \\
\hline & Column Hame & Data Type & Allow Empty \\
\hline B & EmpID & $\operatorname{char}(10)$ & $\square$ \\
\hline & EmpHame & char (10) & $\square$ \\
\hline & EmpSex & char (2) & $\square$ \\
\hline & EmpPosition & char (10) & $\square$ \\
\hline & EmpTel & char (20) & 甲 \\
\hline & EmpPwd & nvarchar (50) & $\square$ \\
\hline & EmpPermissions & nvar char (20) & $\square$ \\
\hline
\end{tabular}

Figure 10. Employee Information Table

Started SQL Server Management Studio Express, then connected the server .After creating database we could get the new user list through the analysis. It would be ok that the data was put into the corresponding list.

IV.

\section{CONCLUSION}

A scientific database structure was established in this paper from our college library information management for practical. Its data redundancy was less, and it had a good data consistency, operability and safety. Combined with the front desk development tools and platform, it can realize the function of strong design and system implementation. It had been showed that library management information system's reliability and stability had been sure through the use of them, which was developed by using the database structure. It had strong practical significance and application value also. At the same time, it had a good reference to design database of management information system.

\section{REFERENCES}

[1] Mengli.The supporting role of digital library in cultivating innovative talents[J].Beijing Forestry University Journal (Social science edition),2004,(S1).

[2] Feisi science and technology R\&D center,SQL Server 2005 Senior management and development [M],Publishing house of electronics industry, 2002

[3] Editor:LiXiangMin,XuJin,JiangShiFeng,etc.SQL Server 2005 Programmer guide $[\mathrm{M}]$,Beijing Hopes electronic publishing house 2001

[4] Sashixuan,Wangshan,An introduction to database system (third edition) Beijing:Advanced Education press,2000

[5] Heguangzhong. A System of Thermal Unit Start-Up (Shut-Down) Cost Based on.NET Technology:[Master degree theses].Wuhan:Huazhong University of Science and Technology,2006.

[6] Thomas Kyte.Effective Oracle by Design:Design and Build High-Performance Oracle Applications. McGraw-Hill.2006,1.233 234 